\title{
Shaken Mannequin Experiments: Head Motion Pattern and Its Potential Effect on Blood Pressure
}

\author{
Srdjan Cirovic, Marco Freddolini, Rachel Goodwin, and Daniel Zimarev
}

The Centre for Biomedical Engineering, University of Surrey, Guildford, Surrey, GU2 7XH, UK

Address correspondence to Srdjan Cirovic, s.cirovic@surrey.ac.uk

Received 5 January 2012; Accepted 10 June 2012

\begin{abstract}
Shaken baby syndrome (SBS) describes a group of symptoms observed in young infants that are associated with a high level of fatality. There is currently no consensus on whether shaking alone can cause the observed injuries. We performed experiments on anthropometric test devices (dummies) to analyze the head motion generated by shaking. Volunteers were asked to violently shake the dummies whose motion was tracked with a camera-based system; a model of the cardiovascular system was mounted on a dummy to assess the effect of shaking on the blood pressure. Over the duration of shaking episodes the acceleration along the inferior-superior axis of the head had a mean of up to four times the gravitational acceleration. The results from the cardiovascular model indicate that this could lead to an increase of the blood pressure in the head. It is possible that this mechanism contributes to some ocular symptoms associated with SBS.
\end{abstract}

Keywords abusive head injury; retinal hemorrhage; acceleration trauma; negative $\mathrm{g}$

\section{Introduction}

Shaken baby syndrome (SBS) is believed to be a form of child abuse which is caused when the victim, a child under two years of age, is violently shaken by the perpetrator [1, $9,10,15]$. Typical symptoms involve subdural and subarachnoid hemorrhage, diffuse axonal injury, and retinal hemorrhage $[1,9,10,13,15]$. The incidence of eye hemorrhage in suspected victims is very high, which is in contrast with accidental head injury $[9,15,17]$. There is a high level of controversy attached to the syndrome, largely because the mechanisms of the observed injuries are not well understood $[3,11,14,16]$. Biomechanical studies in which volunteers vigorously shook models of infants revealed accelerations that were well below the recognized injury threshold levels for impact trauma in adults $[4,6,12]$. However, to our knowledge, there has been no attempt to date to fully quantify three-dimensional head motion pattern generated by shaking which may be important because it could point to injury mechanisms that are very different from those that are typical of high velocity impact accidents. To that end we performed two "shaken mannequin" experiments. In the first experiment we used a camera-based system to track the movement of dummies during shaking trials and to extract kinematic parameters of head motion. As the results of the first experiment revealed that the motion pattern is such that it may affect the blood pressure in the head, we performed the second experiment in which a model of the cardiovascular system was added to a dummy and the pressures in the model were measured while the dummy was shaken.

\section{Methods}

\subsection{Experiment 1}

Two dummies were used in the experiment: a standard P3/4 crash test device (First Technology Safety Systems, Plymouth, USA) and a custom-made simple dummy whose dimensions roughly corresponded to those of a newborn. The smaller custom made device was needed since most victims are younger than six months; while crash test dummies of a newborn were available, they were either made as a single block or had necks that were unrealistically stiff at very low accelerations. The dimensions of the P3/4 dummy are based on the data for a 50th percentile nine month old baby; its overall mass is $9 \mathrm{~kg}$. The custom-made dummy was of simple construction so that it could easily accommodate the cardiovascular model described later in the text. It consisted of only head, neck, and torso. The torso was constructed from a $335 \mathrm{~mm} \times 100 \mathrm{~mm} \times 40 \mathrm{~mm}$ block of wood. The head was represented by a hard plastic ball $92 \mathrm{~mm}$ in diameter and with a mass of $0.48 \mathrm{~kg}$. The neck, which was of silicon rubber, was $40 \mathrm{~mm}$ long and had a diameter of $33 \mathrm{~mm}$. The overall mass was $1.5 \mathrm{~kg}$.

Five male and five female volunteers, 23 to 43 years of age, were asked to hold a dummy at the chest and shake it as violently as they could. The duration of shaking episodes was set at $10 \mathrm{~s}$ for the constructed dummy and $5 \mathrm{~s}$ for the much heavier P3/4 dummy. An array of infrared cameras (Qualisys, Proreflex 500, Qualisys AB, Gothenburg, Sweden) was used to track the position of reflective markers 


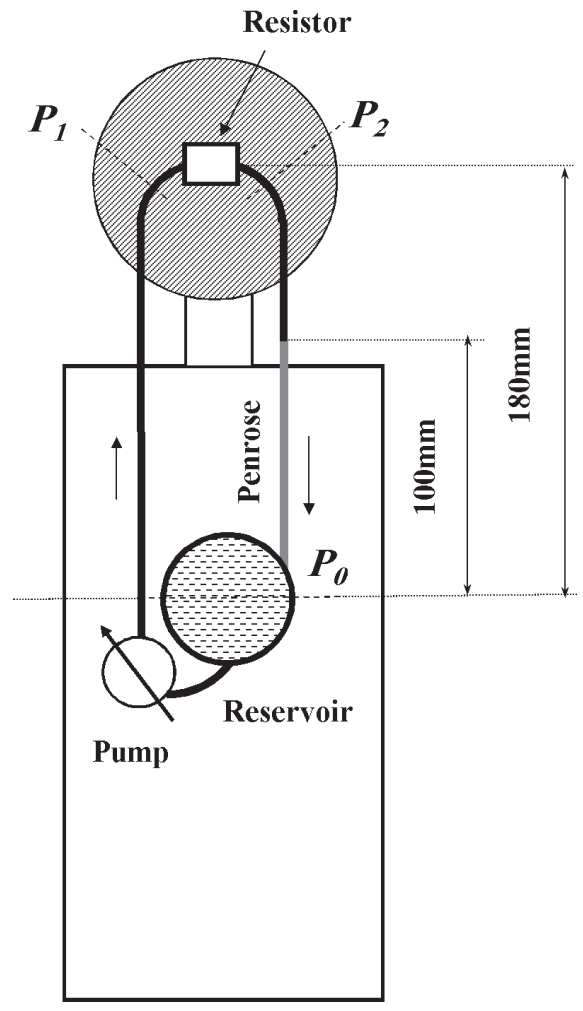

Figure 1: The constructed dummy and the model of the cardiovascular system.

attached to the torso and the head of the dummies at a rate of 250 frames per second. The motion was reconstructed from the filtered marker position data using the associated Qualisys Track Manager software. The raw data for marker coordinates was low-pass filtered at $20 \mathrm{~Hz}$ to eliminate spurious noise. The cut-off frequency was chosen by comparing calculated accelerations with the readings of an accelerometer (Montran MTN/1800, Montran Ltd., Penn Bucks, UK) attached to the torso.

\subsection{Experiment 2}

A single-loop model of the cardiovascular system was constructed and attached to the custom-made dummy. The cardiovascular model consisted of a reservoir (central venous bed), pump (heart), an ascending arm (carotid arteries), resistor (micro-circulation), and a descending arm (veins in the head/neck), as shown in Figure 1. The reservoir consisted of a cylinder of $50 \mathrm{~mm}$ in diameter whose top was sealed with a loose thin membrane (cling-film); this arrangement assured that the pressure in the reservoir was close to atmospheric regardless of the orientation and movement of the dummy, thus reproducing the "venous hydrostatic indifference point" [8]. A miniature diaphragm pump (Hargraves, Mooresville, North Carolina, USA) provided a constant mean flow of $9 \mathrm{~cm}^{3} / \mathrm{s}$. A latex rubber tube $(8 \mathrm{~mm}$ inner diameter, $1.6 \mathrm{~mm}$ wall thickness) was used for the ascending

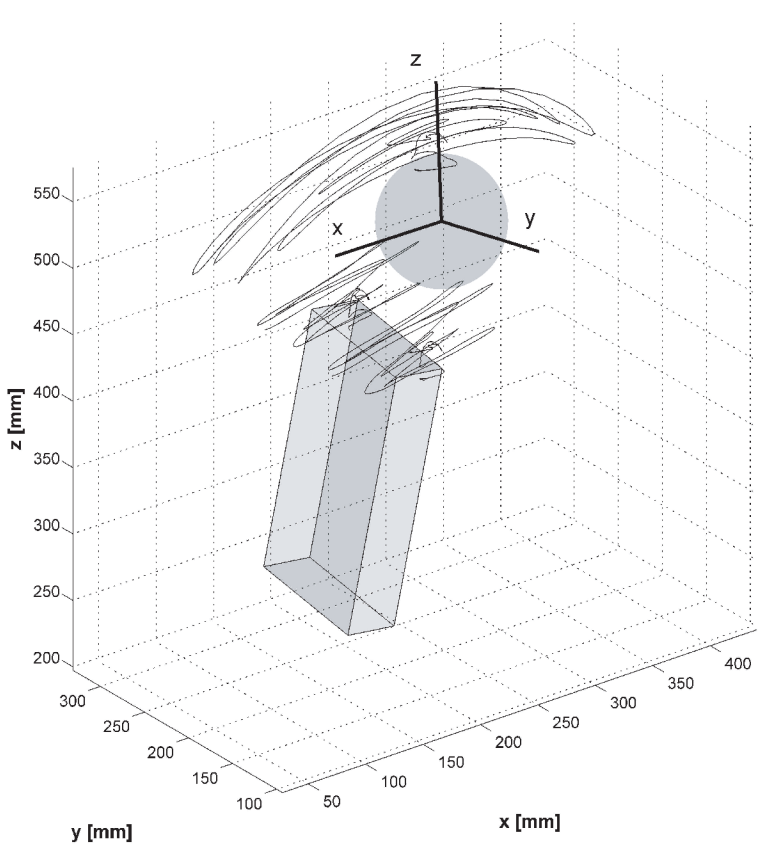

Figure 2: Typical trajectories of the markers attached to the top of the head and the shoulders for the constructed dummy. The coordinates refer to a global (fixed) coordinate system. The volunteers were facing in the positive $\mathrm{x}$ direction. The figure also shows the local (moving) coordinate system of the head.

arm. The resistor was screw-type and it was adjusted to give a pressure drop of approximately $90 \mathrm{mmHg}(1 \mathrm{mmHg} \approx$ $133 \mathrm{~Pa}$ ). The descending arm consisted of a thin-walled Penrose surgical tubing $9.5 \mathrm{~mm}$ in diameter (jugular veins). When the dummy was upright and still, the Penrose tube collapsed and reproduced the "vascular waterfall" effect in which the pressure is uniform along the length of the collapsed jugular vein [7]. Water which has density similar to that of blood was used as a working fluid; low viscosity of water was acceptable since the resistive pressure drop was mainly confined to the resistor. Nine volunteers, six male and three female, between 27 and 45 years of age participated in the experiment. The dummy was held in an upright position and shaken with maximal effort. Pressure was measured with miniature transducers (Piezo pressure sensor, 5 psi range, Honeywell, USA) at the mid-line on the reservoir near its top $\left(P_{0}\right)$, at the resistor just upstream of the constriction $\left(P_{1}\right)$, and at the top of the descending arm $\left(P_{2}\right)$. The pressure signals were digitally low-pass filtered at $20 \mathrm{~Hz}$.

\section{Results and discussion}

\subsection{Experiment 1}

For both devices the head movement occurred mainly in the "sagittal" plane and there was a pronounced whiplash motion of the head (see Figure 2). The dominant frequency of movement was essentially the same for the P3/4 and the 


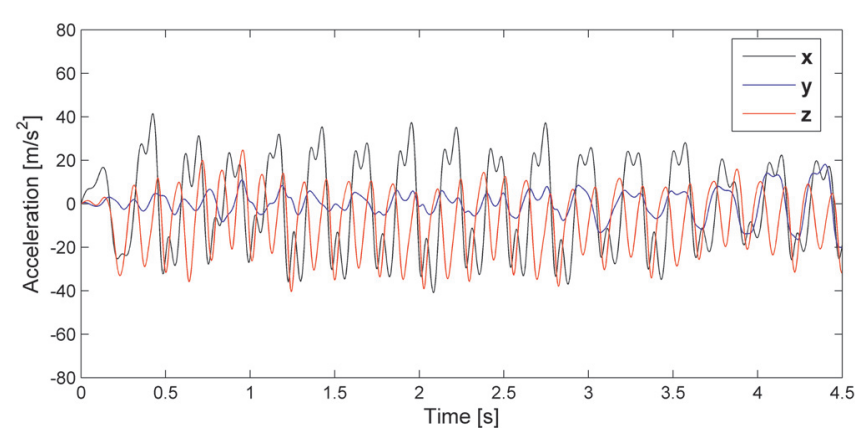

(a) P3/4 dummy.

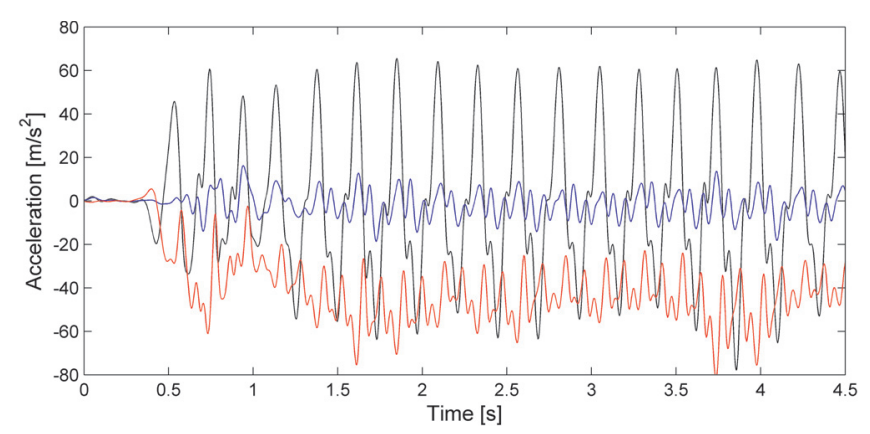

(b) Constructed dummy.

Figure 3: Components of the linear acceleration of the head resolved along the anatomical axes of the head.

Table 1: Peak values of kinematic parameters of head motion averaged across all the volunteers.

\begin{tabular}{lccc}
\hline & $\begin{array}{l}\text { Linear } \\
\text { acceleration }\left[\mathrm{m} / \mathrm{s}^{2}\right]\end{array}$ & $\begin{array}{l}\text { Angular } \\
\text { velocity }\left[\mathrm{s}^{-1}\right]\end{array}$ & $\begin{array}{l}\text { Angular } \\
\text { acceleration }\left[\mathrm{s}^{-2}\right]\end{array}$ \\
\hline P3/4 Dummy & $45 \pm 12$ & $25 \pm 7$ & $650 \pm 180$ \\
\hline $\begin{array}{l}\text { Constructed } \\
\text { Dummy }\end{array}$ & $76 \pm 19$ & $40 \pm 8$ & $1180 \pm 320$ \\
\hline
\end{tabular}

Table 2: Pressure increase over a shaking trial in the cardiovascular model averaged across all the volunteers.

\begin{tabular}{ccc}
\hline & $P_{1}[\mathrm{mmHg}]$ & $P_{2}[\mathrm{mmHg}]$ \\
\hline Mean & $35 \pm 10$ & $25 \pm 10$ \\
\hline Peak & $65 \pm 15$ & $60 \pm 15$ \\
\hline
\end{tabular}

constructed dummy, in spite of the difference in their size and mass; for the whole cohort of volunteers the average value was $3.9 \pm 0.5 \mathrm{~Hz}$ (mean \pm standard deviation) for both dummies. The peak values of kinematic parameters averaged across all of the volunteers are given in Table 1 . The typical traces of the linear acceleration are shown in Figure 3. The accelerations are resolved along the axes of the (moving) coordinate system of the head shown in Figure 2. The most interesting finding was that the $\mathrm{z}$ component of the head acceleration fluctuated about a negative non-zero mean throughout each shaking trial. On the average that mean was $-26 \pm 9 \mathrm{~m} / \mathrm{s}^{2}$ in the case of the constructed dummy and $-6 \pm 3 \mathrm{~m} / \mathrm{s}^{2}$ in the case of the P3/4 dummy. We attribute this result to the constant whiplash motion of the head which involved a centripetal force (acceleration).

\subsection{Experiment 2}

Figure 4 displays results for the pressures in the model which appeared consistently in all of the shaking trials. While $P_{0}$ remained close to zero and experienced only moderate fluctuations, $P_{1}$ and $P_{2}$ mirrored the profile of head $\mathrm{z}$ acceleration documented in the first experiment. Thus, the mean values of both pressures were elevated from their respective base values throughout the duration of shaking. These two pressures also experienced large amplitude oscillations with a dominant frequency of about $4 \mathrm{~Hz}$. The results for mean and peak increases in $P_{1}$ and $P_{2}$ averaged across all experimental trials are summarized in Table 2.

The magnitudes of the linear and angular accelerations for the head are consistent with the lower end of the results reported by previous similar studies $[6,12]$. The main novel finding is that the whiplash motion of the head produced $\mathrm{z}$ component of head acceleration which had a non-zero mean throughout a shaking trial. This pattern of head movement was observed in both dummies, in spite of them being very different in terms of overall mass and construction of neck, and should therefore be inherent to the shaking motion. To our knowledge, this observation has not been reported before in the open literature. One possible reason for that is that many of the previous studies focused on the tangential ( $x$ ) component of head acceleration. The other possible reason is that acceleration measuring devices had poor response at very low frequencies and thus could not register the "steady state" component of the head acceleration. A potential consequence of the observed acceleration pattern is the pooling of blood towards the head. This hypothesis is supported by the results of the second experiment.

\section{Conclusions}

When the body is accelerated, the inertia of blood adds a hydrostatic pressure gradient in the direction opposite to that of the acceleration vector. Thus, accelerating in the feet-tohead direction at $1 \mathrm{~g}$ (where $g=9.81 \mathrm{~m} / \mathrm{s}^{2}$ is the acceleration due to gravity) has the effect of doubling the weight of the blood column in the major blood vessels. This is commonly referred to as exposure to " $+2 \mathrm{~g}$ ". On the other hand, an acceleration in the head-to-feet direction can cause the effect of "negative g" in which the pressure increases from the heart towards the head. The effects of negative $g$ have been investigated in the context of high performance aircraft maneuvers and emergency ejections $[2,8]$. It has been shown 


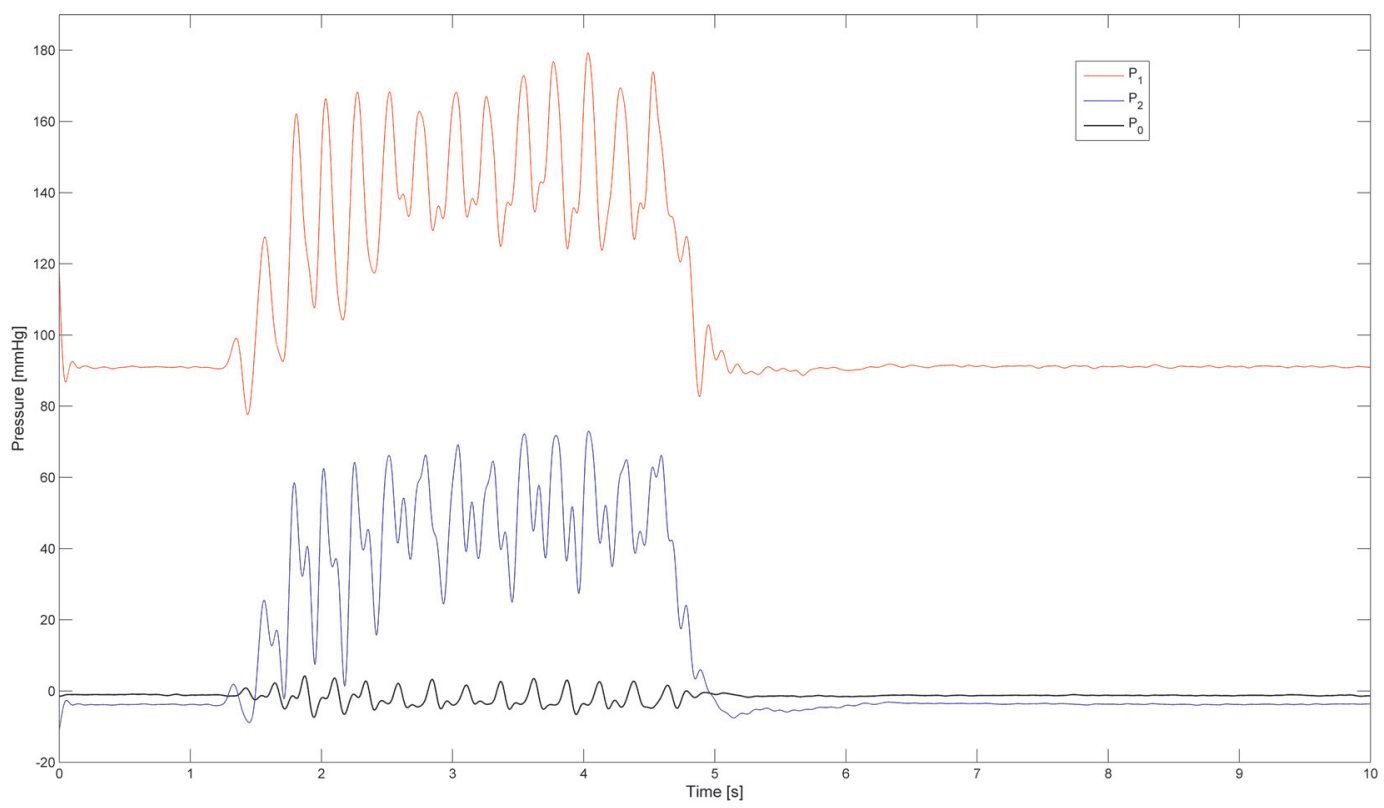

Figure 4: Typical traces of pressure recorded at the "eye level" and the "heart level" of the cardiovascular model during a shaking trial.

that in adults exposure to $-3 \mathrm{~g}$ for $10 \mathrm{~s}$ or exposure to $-7 \mathrm{~g}$ for $1 \mathrm{~s}$ may cause eye hemorrhages via an elevated venous pressure in the head [2,5]. Our results suggest that it is feasible that vigorous shaking could create the negative $\mathrm{g}$ effect of substantial magnitude. While this potential mechanism is not likely to contribute to the brain injuries leading to fatalities, it may contribute to eye hemorrhaging that is frequently found in the suspected cases of SBS. We therefore feel that this idea merits further attention in the future.

Acknowledgments The authors would like to express their gratitude to the staff of the Transportation Research Laboratory, Berkshire, UK for kindly agreeing to provide the P3/4 anthropometric device used in this study.

\section{References}

[1] I. Blumenthal, Shaken baby syndrome, Postgrad Med J, 78 (2002), 732-735.

[2] V. A. Byrnes, Elevated intravascular pressure as an etiologic mechanism in the production of eye injuries, Trans Am Ophthalmol Soc, 57 (1959), 473-538.

[3] M. E. Case, Abusive head injuries in infants and young children, Leg Med, 9 (2007), 83-87.

[4] C. Z. Cory and B. M. Jones, Can shaking alone cause fatal brain injury? A biomechanical assessment of the Duhaime shaken baby syndrome model, Med Sci Law, 43 (2003), 317-333.

[5] D. B. David, T. Mears, and M. P. Quinlan, Ocular complications associated with bungee jumping, Br J Ophthalmol, 78 (1994), 234-235.

[6] A. C. Duhaime, T. A. Gennarelli, L. E. Thibault, D. A. Bruce, S. S. Margulies, and R. Wiser, The shaken baby syndrome. A clinical, pathological, and biomechanical study, J Neurosurg, 66 (1987), 409-415.

[7] A. C. Guyton and J. E. Hall, Textbook of Medical Physiology, WB Saunders Company, Philadelphia, PA, 9th ed., 1996.
[8] P. Howard, The physiology of positive acceleration, in A Textbook of Aviation Physiology, J. A. Gillies, ed., Pergamon Press, Oxford, 1965, 551-687.

[9] J. Matschke, B. Herrmann, J. Sperhake, F. Körber, T. Bajanowski, and M. Glatzel, Shaken baby syndrome: a common variant of non-accidental head injury in infants, Dtsch Arztebl Int, 106 (2009), 211-217.

[10] J. Matschke, K. Püschel, and M. Glatzel, Ocular pathology in shaken baby syndrome and other forms of infantile nonaccidental head injury, Int J Legal Med, 123 (2009), 189-197.

[11] M. C. Pierce and G. Bertocci, Injury biomechanics and child abuse, Annu Rev Biomed Eng, 10 (2008), 85-106.

[12] M. T. Prange, B. Coats, A. C. Duhaime, and S. S. Margulies, Anthropomorphic simulations of falls, shakes, and inflicted impacts in infants, J Neurosurg, 99 (2003), 143-150.

[13] W. Squier, Shaken baby syndrome: the quest for evidence, Dev Med Child Neurol, 50 (2008), 10-14.

[14] D. G. Talbert, Shaken baby syndrome: does it exist?, Med Hypotheses, 72 (2009), 131-134.

[15] B. M. Togioka, M. A. Arnold, M. A. Bathurst, S. M. Ziegfeld, R. Nabaweesi, P. M. Colombani, et al., Retinal hemorrhages and shaken baby syndrome: an evidence-based review, J Emerg Med, 37 (2009), 98-106.

[16] B. Wilkins, Head injury—abuse or accident?, Arch Dis Child, 76 (1997), 393-396.

[17] T. Wygnanski-Jaffe, A. V. Levin, A. Shafiq, C. Smith, R. W. Enzenauer, J. E. Elder, et al., Postmortem orbital findings in shaken baby syndrome, Am J Ophthalmol, 142 (2006), 233-240. 